\title{
Particle swarm optimization tuned fuzzy terminal sliding mode control for UPS inverters
}

\author{
En-Chih Chang ${ }^{\mathrm{a}}$, Zhiyi Su ${ }^{\mathrm{b}}$, Ziang $\mathrm{Xu}^{\mathrm{a}}$ and Rong-Ching $\mathrm{Wu}^{\mathrm{a}, *}$ \\ ${ }^{a}$ Department of Electrical Engineering, I-Shou University, Dashu District, Kaohsiung City, Taiwan, R.O.C. \\ ${ }^{\mathrm{b}}$ School of Control Science and Engineering of Shandong University, Shanda Nanlu, Jinan, P.R.C.
}

\begin{abstract}
This paper proposes a particle swarm optimization algorithm tuned fuzzy terminal sliding mode control for the application of UPS inverters. Though classic sliding mode control (SMC) is insensitive to system uncertainties, it possesses an infinite system-state convergence time. For high-accuracy tracking control, a terminal sliding mode control (TSMC) is developed to provide a finite system-state convergence time. However, difficult estimation occurs in TSMC, and incurs high UPS inverter voltage harmonics and slow dynamic response. To obtain high-quality UPS inverter output voltage, a fuzzy logic (FL) with a computationally simple and practically easy estimator is integrated into TSMC to resolve system uncertainties. Simultaneously, the particle swarm optimization (PSO) algorithm is applied to optimally tune the control gains of the TSMC with a fuzzy estimator. Results indicate that the presented combination of PSO, FL and TSMC yields a closed-loop UPS inverter with good performance under various loading conditions. Simulation and experimental results indicate that the proposed control can achieve low total harmonic distortion (THD) under nonlinear loading conditions and fast dynamic response under transient loading conditions.
\end{abstract}

Keywords: Terminal sliding mode control (TSMC), finite system-state convergence time, fuzzy logic (FL), particle swarm optimization (PSO), total harmonic distortion (THD)

\section{Introduction}

Uninterruptible power supply (UPS) has been widely applied to provide emergency power to critical loads including safe lighting, telecommunication centers and life support equipment in hospitals that cannot afford utility failures [2]. In UPS systems, the overall performance is dependent upon the static inverter-filter arrangement, which is used to convert DC voltage to a sinusoidal AC output. A primary concern of a UPS inverter is to determine an effective controller which

\footnotetext{
${ }^{*}$ Corresponding author. Rong-Ching Wu, Department of Electrical Engineering, I-Shou University, No.1, Sec. 1, Syuecheng Rd., Dashu District, Kaohsiung City 84001, Taiwan, R.O.C. Tel.: +886 76577711 (Ext: 6636); Fax: +886 76577205; E-mail: rcwu@isu. edu.tw.
}

is able to achieve accurate tracking of the desired sinewave command. Accurate tracking for a UPS inverter should meet the following requirements: 1) perfect sinusoidal voltage waveform, even under nonlinear loading; 2) fast transient response under sudden load changes; 3 ) a steady-state error of approximately zero. Owing to the simple structure and easy design, a PID control is typically used as the control method for the inverter. However, a PID controller cannot achieve satisfactory performance when the controlled plant experiences highly nonlinear loading [5, 6]. Several control methods have been employed, such as H-infinity control, repetitive control, and multi-loop control, but they are difficult to realize, and their algorithms are extremely complex $[4,7,11]$. Sliding mode control (SMC) is intrinsically robust against system 
uncertainties [15]. The SMC has been presented as a good alternative for UPS inverter design, but a linear sliding surface is employed and the system trajectory has an infinite system-state convergence time $[12,13]$. Recently, a terminal sliding mode control (TSMC) with a nonlinear sliding-surface has been developed and applied to many fields. Compared to linear slidingsurface-based control, the TSMC can force the system tracking error to converge to zero in finite time, and thus achieve a high-accuracy tracking control $[9,14]$. This paper introduces such a control which possesses a finite system-state convergence time mechanism to overcome the disadvantages of classic SMC with an infinite system-state convergence time for special application to UPS inverters. However, the estimation of system uncertainties is a difficult problem in TSMC systems. Such problems will yield high THD, nonzero steady-state errors, and low dynamic responses in the terminal sliding mode controlled UPS inverter. Fuzzy logic (FL) controllers have been applied to a wide range of applications in various fields. FL has been further developed for power conversion applications, since its operation principle does not require precise knowledge of the load parameters $[1,3]$. Hence, the estimation of system uncertainties can be accomplished by FL. The particle swarm optimization (PSO) algorithm is also an excellent evolutionary algorithm, and has been widely applied to many areas of science and engineering $[8,10]$. To enhance the performance of UPS inverters, the PSO is used to optimally tune control gains of the TSMC with the fuzzy estimator. By combining PSO, FL, and TSMC, the presented system will easily achieve finite system-state convergence time, a zero steady-state error, fast dynamic response, and prevent a low THD under various loading conditions. Simulation and experimental results are presented to illustrate the good performance of the proposed controlled UPS inverter.

\section{Mathematical modeling of UPS inverter}

As shown in Fig. 1, the output voltage $v_{o}$ of the UPS inverter can be forced to track a sinusoidal reference voltage, $v_{d}$ by applying the proposed control.

As shown in Fig. 1, two equations can be obtained by the use of the KVL and KCL:

$$
i_{L}=i_{c}+i_{o}=C \frac{d v_{o}}{d t}+\frac{v_{o}}{R}
$$

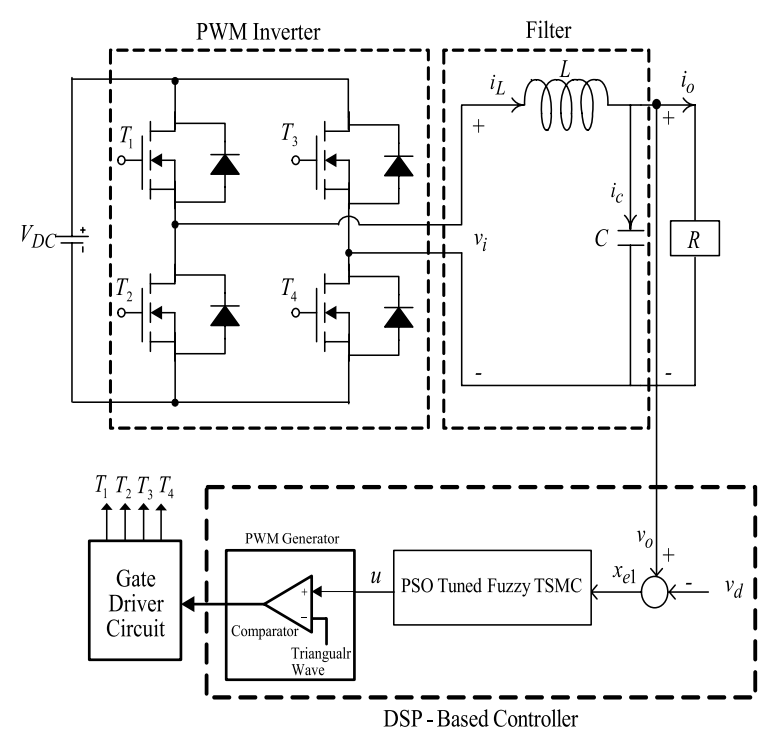

Fig. 1. Digitally controlled UPS inverter.

$$
L \frac{d i_{L}}{d t}+v_{o}=v_{i}
$$

Substituting Equations (1) into (2) yields the following:

$$
\frac{d^{2} v_{o}}{d t^{2}}+\frac{1}{R C} \frac{d v_{o}}{d t}+\frac{1}{L C} v_{o}=\frac{1}{L C} v_{i}
$$

Let $\quad x_{1}=v_{o}, \quad x_{2}=\dot{x}_{1}=\dot{v}_{o}, \quad \dot{x}_{2}=\ddot{x}_{1}=\ddot{v}_{o}$ and $u=v_{i}$. Then, the matrix equation can be expressed as:

$$
\left[\begin{array}{l}
\dot{x}_{1} \\
\dot{x}_{2}
\end{array}\right]=\left[\begin{array}{cc}
0 & 1 \\
-\frac{1}{L C} & -\frac{1}{R C}
\end{array}\right]\left[\begin{array}{l}
x_{1} \\
x_{2}
\end{array}\right]+\left[\begin{array}{c}
0 \\
\frac{1}{L C}
\end{array}\right] u
$$

Suppose the desired output voltage is $x_{d}=v_{d}$, and a state variable $x_{e}=\left[\begin{array}{ll}x_{e 1} & x_{e 2}\end{array}\right]^{T}$ related to the tracking error is expressed by new state variables as follows:

$$
\begin{aligned}
x_{e 1}= & x_{1}-x_{d}=v_{o}-v_{d} \\
x_{e 2}= & \dot{x}_{e 1}=\dot{x}_{1}-\dot{x}_{d}=\dot{v}_{o}-\dot{v}_{d} \\
\ddot{x}_{e 2}= & -\frac{1}{L C} x_{e 1}-\frac{1}{R C} x_{e 2}+\frac{1}{L C} u \\
& -\frac{1}{L C} v_{d}-\frac{1}{R C} \dot{v}_{d}-\ddot{v}_{d}
\end{aligned}
$$

Thus, the error state matrix can be obtained as 


$$
\begin{gathered}
{\left[\begin{array}{c}
\dot{x}_{e 1} \\
\dot{x}_{e 2}
\end{array}\right]=\left[\begin{array}{cc}
0 & 1 \\
-a_{1}-a_{2}
\end{array}\right]\left[\begin{array}{l}
x_{e 1} \\
x_{e 2}
\end{array}\right]+\left[\begin{array}{c}
0 \\
a_{1}
\end{array}\right] u} \\
+\underbrace{\left[-a_{1} v_{d}-a_{2} \dot{v}_{d}-\ddot{v}_{d}\right.}_{w}]
\end{gathered}
$$

where $a_{1}$ indicates $1 / L C, a_{2}$ is $1 / R C$, and $w$ denotes the interference. Thus, our purpose is to design a control law $u$. Once the $u$ is accurately designed, the output of the UPS inberter will remain constant at the desired $v_{d}$.

\section{Control design}

For the sake of brevity, system (6) is redefined as follows:

$$
\begin{aligned}
& \dot{e}_{1}=e_{2} \\
& \dot{e}_{2}=f\left(e_{1}, e_{2}\right)+b\left(e_{1}, e_{2}\right) u(t)
\end{aligned}
$$

where $e_{1}$ and $e_{2}$ represent the system error states, $f\left(e_{1}, e_{2}\right)$ and $b\left(e_{1}, e_{2}\right)$ are nonlinear functions of $e_{1}$ and $e_{2}$, and $u(t)$ is the control input.

The finite-time convergent sliding function is designed as follows:

$$
s=\dot{\sigma}_{0}+\beta_{1} \sigma_{0}^{\gamma_{1}}
$$

where $\sigma_{0}=e_{1}$ and $\beta_{1}$ is constant.

From Equation (8):

$$
\begin{aligned}
\dot{s} & =\ddot{\sigma}_{0}+\beta_{1} \gamma_{1} \sigma_{0}^{\gamma_{1}-1} \dot{\sigma}_{0} \\
& =-\sum_{i=1}^{2} a_{i} e_{i}+b u-w(t)+\beta_{1} \gamma_{1} \sigma_{0}^{\gamma_{1}-1} \dot{\sigma}_{0}
\end{aligned}
$$

Define

$$
\begin{aligned}
& a_{i}=a_{i 0}+\Delta a_{i}, i=1,2 \\
& b=b_{0}+\Delta b \\
& w(t)=a_{1} v_{d}+a_{2} \dot{v}_{d}+\ddot{v}_{d}
\end{aligned}
$$

where $a_{i 0}$ denotes the nominal value of $a_{i}, b_{0}$ represents the nominal value of $b, \Delta a_{i}$ indicates the variation of $a_{i}$, and $w(t)$ is the interference.

For system (6), the control law $u$ can be expressed as follows:

$$
u=u_{e}+u_{f t}
$$

where the equivalent control term $u_{e}$ is valid only on the sliding surface, and is defined as follows:

$$
\begin{array}{r}
\left.\dot{s}\right|_{u=u_{e}, a_{1}=a_{10}, a_{2}=a_{20}, b=b_{0}, w(t)=0}=0 \\
\therefore u_{e}=\left(\sum_{i=1}^{2} a_{i 0} e_{i}-\beta_{1} \gamma_{1} \sigma_{0}^{\gamma_{1}-1} \dot{\sigma}_{0}\right) / b_{0}
\end{array}
$$

While the system dynamics are in sliding action, $s=0$ :

$$
\dot{\sigma}_{0}=-\beta_{1} \sigma_{0}^{\gamma_{1}}
$$

Substituting Equations (14) into (13) yields the following:

$$
u_{e}=\left(\sum_{i=1}^{2} a_{i 0} e_{i}+\beta_{1}^{2} \gamma_{1} \sigma_{0}^{2 \gamma_{1}-1}\right) / b_{0}
$$

Let

$$
u_{f t}=\sum_{i=1}^{2} \phi_{i} e_{i}+\sum_{i=1}^{2} \psi_{i} \sigma_{0}^{2 \gamma_{1}-1}+N
$$

where the $u_{f t}$ guarantees the existence of the finitetime sliding mode, and is used to eliminate system uncertainties.

The condition for the existence of a sliding motion is, $s \dot{s}<0$ as follows:

$$
\begin{aligned}
\left.s \dot{s}\right|_{u=} & u_{e}+u_{f t}=\left\{-\sum_{i=1}^{2} \Delta a_{i} e_{i}-\Delta a_{2} e_{2}\right. \\
& +\left[\frac{\Delta b}{b_{0}}\left(\sum_{i=1}^{2} a_{i 0} e_{i}\right)\right]+\left[b\left(\sum_{i=1}^{2} \phi_{i} e_{i}\right)\right] \\
& +\left(b \psi_{1}-\frac{\Delta b}{b_{0}} \beta_{1}^{2} \gamma_{1}\right) \sigma_{0}^{2 \gamma_{1}-1} \\
& +\left(-\beta_{1}^{2} \gamma_{1} \sigma_{0}^{2 \gamma_{1}-1}+b \psi_{2} \sigma_{0}^{2 \gamma_{1}-1}\right) \\
& +[b N-w(t)]\} s<0
\end{aligned}
$$

The conditions $\phi_{i}, \psi_{i}$ and $N$ to satisfy Equation (17) can next be obtained. Notice that $w(t)$ in Equation (17) is an interference and in practice should be rewritten as $w(k)$, where $k$ denotes the sample interval. Because $w(k)$ is generally uncertain, the control in this form cannot be implemented and must be estimated.

Therefore, define the uncertainty estimation error $\tilde{w}_{e}(k)$ as follows:

$$
\tilde{w}_{e}(k)=w(k)-\hat{w}(k)
$$


Then, an algorithm is expressed as:

$$
\begin{gathered}
\hat{w}(k)=\hat{w}(k-1)+\beta(\hat{w}(k-1)-\hat{w}(k-2)) \\
+\tilde{w}_{e}(k-1)+\beta\left(\tilde{w}_{e}(k-1)-\tilde{w}_{e}(k-2)\right)
\end{gathered}
$$

where $\hat{w}(k-1)$ is the estimated value of $w(k-1)$; the subscript indicates $(k-1)$ and $(k-2)$ representing the $(k-1)$ th and $(k-2)$ th sampling intervals, respectively; and $|\beta| \leq 1$ is an adjustable parameter.

The FL is used to tune the $\beta$ in Equation (19), and the fuzzy rule can be expressed as a two-input single-output system as follows:

IF $\tilde{w}_{e}(k-1)$ is $\tilde{W}$ and $\Delta \tilde{w}_{e}(k-1)$ is $\Delta \tilde{W}$

THEN $\beta$ is beta

where $\tilde{W}, \Delta \tilde{W}$, and beta are the fuzzy sets of $\tilde{w}_{e}(k-1)$, $\Delta \tilde{w}_{e}(k-1)$, and $\beta$, respectively.

The $\tilde{w}_{e}(k-1)$ and $\Delta \tilde{w}_{e}(k-1)$ can be easily obtained according to the following processes.

The incremental change of $\tilde{w}_{e}(k-1)$ is defined as follows:

$$
\Delta \tilde{w}_{e}(k-1)=\tilde{w}_{e}(k-1)-\tilde{w}_{e}(k-2)
$$

From Equations (18) through (21), when the parameter $\beta$ is well tuned, the $\tilde{w}_{e}(k-1)$ and $\Delta \tilde{w}_{e}(k-1)$ will be forced to zero and the $\tilde{w}_{e}(k)$ will tend toward zero. Thus, the system dynamics of system (6) will be insensitive to system perturbations, and the closed-loop system can stabilize asymptotically. The resulting fuzzy rule base designed with a two-dimensional phase plane for tuning $\beta$ is displayed in Table 1 .

Though the control gains can be tuned by the use of $\phi_{i}, \psi_{i}$ and $N$, thus achieving finite system-state convergence time, Equation (16) implies that the sine function across the surface $s$, and therefore chatter phenomenon,

Table 1

Fuzzy rule base

\begin{tabular}{|c|c|c|c|c|c|c|c|c|}
\hline \multicolumn{1}{|c|}{} & \multicolumn{7}{|c|}{$\widetilde{w}_{e}(k-1)$} \\
\cline { 2 - 9 }$\Delta \sqrt{5} \tilde{w}_{e}(k-1)$ & NB & NM & NS & ZE & PS & PM & PB \\
\hline \multirow{5}{*}{} & PB & ZE & NS & NS & NM & NM & NB & NB \\
\cline { 2 - 9 } & PM & PS & ZE & NS & NS & NM & NM & NB \\
\cline { 2 - 9 } & PS & PS & PS & ZE & NS & NS & NM & NM \\
\cline { 2 - 9 } & NS & PM & PS & PS & ZE & NS & NS & NM \\
\cline { 2 - 9 } & NM & PB & PM & PM & PS & PS & ZE & NS \\
\cline { 2 - 9 } & NB & PB & PB & PM & PM & PS & PS & ZE \\
\hline
\end{tabular}

exists. Thus, to eliminate the chatter, the PSO algorithm represented in Equations (22) and (23) is used to optimally tune the control gains of the fuzzy TSMC. Equations (22) and (23) indicate the evolution models of a particle; then, the speed and position of each particle can be updated when moving toward a destination.

$$
\begin{gathered}
V_{i+1}=c_{0} V_{i}+c_{1} \lambda_{1}\left(X_{i}^{\text {pbest }}-X_{i}\right) \\
+c_{2} \lambda_{2}\left(X_{i}^{\text {gbest }}-X_{i}\right) \\
X_{i+1}=X_{i}+V_{i+1}
\end{gathered}
$$

where $c_{0}, c_{1}$ and $c_{2}$ denote variables, $\lambda_{1}$ and $\lambda_{2}$ are random numbers, $V_{i}$ represents the present velocity, $X_{i}$ is the present position, $X_{i}^{\text {pbest }}$ shows the best localized position, and $X_{i}^{\text {gbest }}$ is the best global position. The operation of the PSO algorithm is described as follows. First, define the number of particles, and initialize their speeds and positions. Then, calculate the fitness of each particle according to $F=\sum_{k=1}^{M}\left|v_{o}-v_{d}\right|$. For each particle, compare its fitness with its present best fitness. When the former is better than the latter, its present best fitness and best position are updated by its fitness and present position, respectively. Similarly, for each particle, compare its fitness with the global best fitness of the swarm. When the former is better than the latter, the global best fitness and global best position are updated by the former and the best position of the compared particle, respectively. Then, update the position and speed of each particle according to Equations (22) and (23). Finally, repeat this procedure until the terminal condition is completed.

\section{Simulation and experimental results}

The system parameters are listed as follows: DC source voltage, $V_{D C}=220 \mathrm{~V}$; switching frequency, $f_{s}=18 \mathrm{kHz}$; output voltage and frequency, $v_{o}=110$ $\mathrm{V}_{\mathrm{rms}}, f=60 \mathrm{~Hz}$; full resistive load, $R=12 \Omega$; diode bridge rectifier load (electrolytic capacitor: $200 \mu \mathrm{F}$ and load resistor: $55 \Omega$ ); filter inductor, $L=1.2 \mathrm{mH}$; filter capacitor, $C=10 \mu \mathrm{F}$. Figure 2(a) and (b) depict the simulated output voltage and the load current obtained using the proposed control and the classic SMC, respectively, under TRIAC load (from no load to full load) with a firing angle of $90^{\circ}$ each half cycle. As shown by the figures, the proposed control indicates a smaller voltage drop and a fast recovery of the steady-state response (\%THD of the output voltage equals $1.21 \%$ ), 


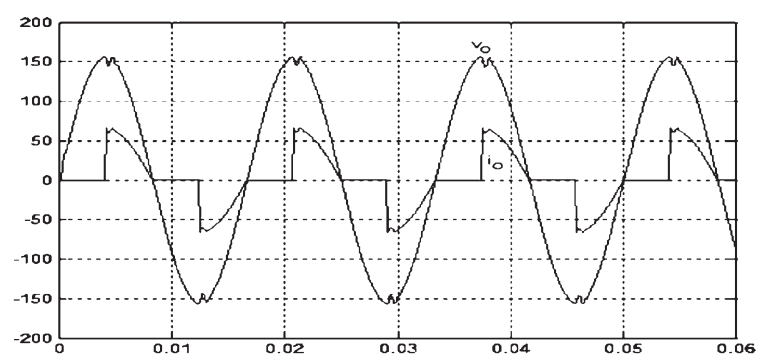

(a) $(50 \mathrm{~V} / \mathrm{div} ; 12 \mathrm{~A} / \mathrm{div})$.

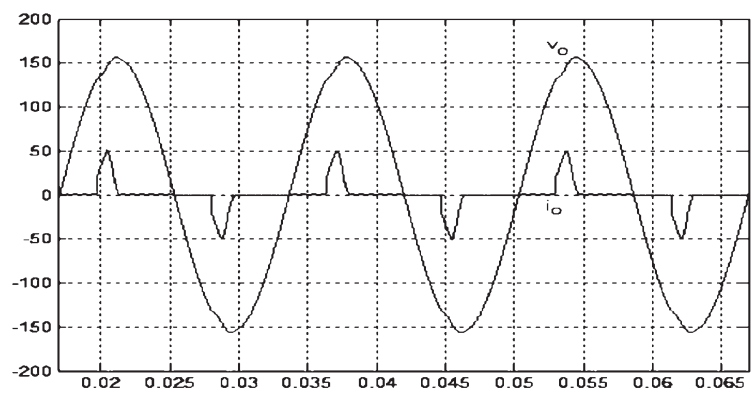

(c) $(50 \mathrm{~V} / \mathrm{div} ; 15 \mathrm{~A} / \mathrm{div})$.

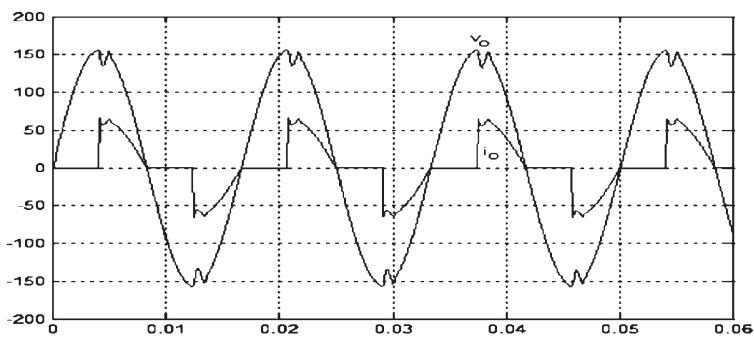

(b) $(50 \mathrm{~V} / \mathrm{div} ; 12 \mathrm{~A} / \mathrm{div})$.

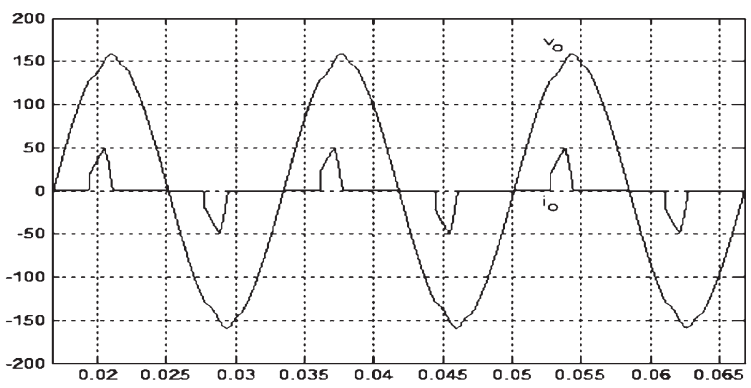

(d) $(50 \mathrm{~V} / \mathrm{div} ; 15 \mathrm{~A} / \mathrm{div})$.

Fig. 2. Simulated waveforms of output voltage and the load current under (a) TRIAC load with the proposed control; (b) TRIAC load with classic SMC; (c) rectifier load with the proposed control; (d) rectifier load with classic SMC.

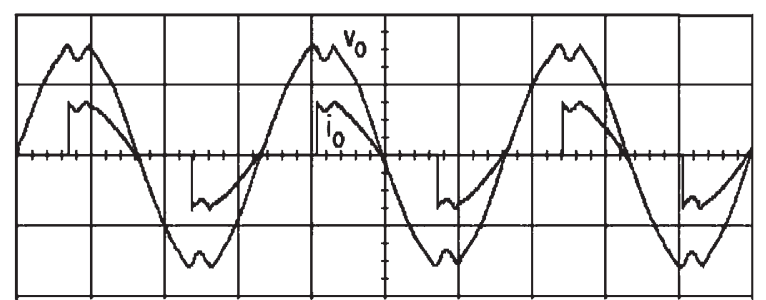

(a) $(100 \mathrm{~V} / \mathrm{div} ; 20 \mathrm{~A} / \mathrm{div} ; 5 \mathrm{~ms} / \mathrm{div})$

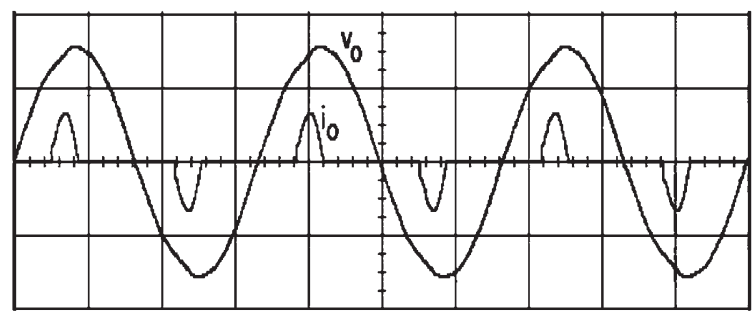

(c) $(100 \mathrm{~V} / \mathrm{div} ; 20 \mathrm{~A} / \mathrm{div} ; 5 \mathrm{~ms} / \mathrm{div})$

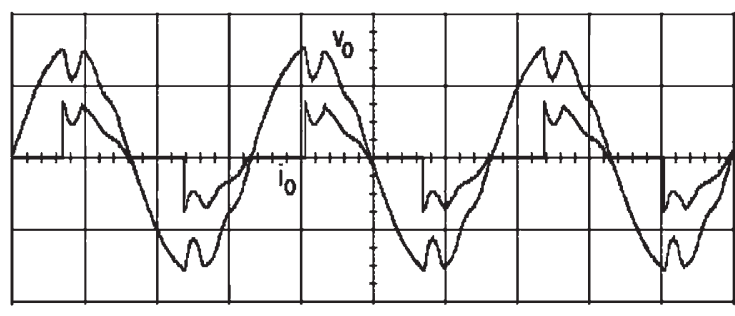

(b) $(100 \mathrm{~V} / \mathrm{div} ; 20 \mathrm{~A} / \mathrm{div} ; 5 \mathrm{~ms} / \mathrm{div})$

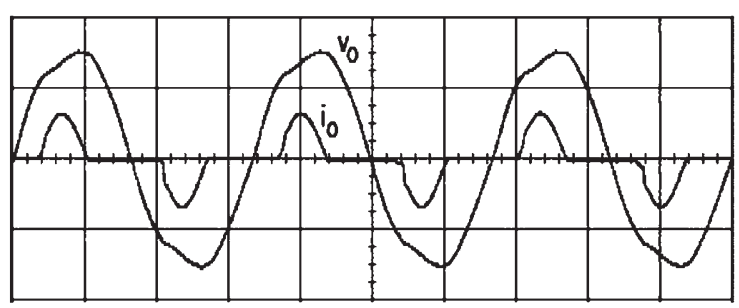

(d) $(100 \mathrm{~V} / \mathrm{div} ; 20 \mathrm{~A} / \mathrm{div} ; 5 \mathrm{~ms} / \mathrm{div})$

Fig. 3. Experimental waveforms of the output voltage and the load current under (a) TRIAC load with the proposed control. (b) TRIAC load with the classic SMC. (c) Rectifier load with the proposed control. (d) Rectifier load with the classic SMC.

as compared to the classic SMC (\% THD of the output voltage equals $2.62 \%$ ). The performance of the inverter with the proposed control under rectifier load is reported. Figure 2(c) indicates that the simulated output voltage waveform is nearly sinusoidal and has very small distortion (\% THD of the output voltage equals $1.30 \%$ ), which exceeds the industrial standard of $5 \%$. Compared to the proposed control, the simulated 
waveforms obtained using the classic SMC under a rectifier load, are reported in Fig. 2(d), with a \%THD of $5.66 \%$; note that the output voltage distortion is high. Additionally, experimental waveforms under TRIAC load are investigated. Figure 3(a) shows the experimental waveform with the proposed control under TRIAC load (from no load to full load) with a firing angle of $90^{\circ}$ each half cycle. Figure 3(b) shows the experimental waveform with the classic SMC under the same load condition. Results indicate that the proposed control provides better compensation of output voltage waveform (i.e., a smaller voltage drop and faster recovery time), particularly at the firing angle (\% THD of the output voltage equals $1.28 \%$ ), as compared to the classic SMC (\% THD of the output voltage equals $2.94 \%$ ). Figure 3(c) and (d) show the experimental output voltage and the load current waveforms of the UPS inverter controlled by the proposed control and the classic SMC under rectifier load, consisting of a full wave rectified with a parallel resistor and capacitor filter. As shown in Fig. 3(c), only a slight distortion (\% THD of the output voltage equals $1.36 \%$ ) occurs in the output voltage when the current rises abruptly. In contrast to the proposed control, the experimental output-voltage waveform obtained using the classic SMC exhibits a high \%THD of $5.87 \%$. Indeed, the proposed control decreases the high THD, reduces the steady-state error, and quickly converges to the origin.

\section{Conclusions}

By combining PSO, FL, and TSMC, the presented system has improved the steady-state and dynamic response of the UPS inverter. The TSMC can resolve the classic SMC problem, but the difficult estimation of system uncertainties still exists in TSMC. Such difficulty may cause high voltage harmonics, and slow transient response. The PSO algorithm is used to optimally tune the control gains of the fuzzy TSMC, thus obtaining robust UPS inverter performance. Simulation and experimental results show that THD and dynamic response results from a UPS inverter under the proposed system exceed the results achieved under the classic SMC system with both linear and nonlinear loading.

\section{Acknowledgments}

This work was supported by the Ministry of Science and Technology of Taiwan, R.O.C., under contract number MOST104-2221-E-214-011.

\section{References}

[1] A. Chikh, and A. Chandra, An optimal maximum power point tracking algorithm for PV systems with climatic parameters estimation, IEEE Trans on Sustainable Energy 6(2) (2015), 644-652.

[2] B.M. Wilamowski and J.D. Irwin, Power electronics and motor drives, CRC Press, Boca Raton, FL, USA, 2011.

[3] B. Radim and J.K. George, Concepts and fuzzy logic, MIT Press, Cambridge, Massachusetts, London, England, 2011.

[4] B. Zhang, K.L. Zhou and D.W. Wang, Multirate repetitive control for PWM DC/AC converters, IEEE Trans on Industrial Electronics 61(6) (2014), 2883-2890.

[5] H.B. Shin and J.G. Park, Anti-windup PID controller With integral state predictor for variable-Speed motor drives, IEEE Trans on Industrial Electronics 59(3) (2012), 1509-1516.

[6] I. Rasoanarivo and F. Sargos, Multi-objective analysis for designing and controlling micro-grids under multi-control with PID, MHCC and FOPID controllers, Proc IEEE Int Conf Industry Applications Society Annual Meeting, 2013, pp. 1-8.

[7] J. He and Y.W. Li, Generalized closed-loop control schemes with embedded virtual impedances for voltage source converters with LC or LCL filters, IEEE Trans on Power Electronics 27(4) (2012), 1850-1861.

[8] K.E. Parsopoulos and M.N. Vrahatis, Particle swarm optimization and intelligence: Advances and applications, information science reference, Hershey, PA, USA, 2010.

[9] K.F. Lu and Y.Q. Xia, Finite-time fault-tolerant control for rigid spacecraft with actuator saturations, IET Proceedings-Control Theory and Applications 7(11) (2013), 1529-1539.

[10] K. Ishaque and Z. Salam, A deterministic particle swarm optimization maximum power point tracker for photovoltaic system under partial shading condition, IEEE Trans on Industrial Electronics 60(8) (2013), 3195-3206.

[11] M. Howlader, N. Urasaki, A. Yona, T. Senjyu and A.Y. Saber, Design and implement a digital $\mathrm{H}$-infinty robust controller for a MW-class PMSG-based grid-interactive wind energy conversion system, Energies 6 (2013), 2084-2109.

[12] P. Sanchis, A. Ursaea, E. Gubia and L. Marroyo, Boost DCAC inverter: A new control strategy, IEEE Trans on Power Electronics 20(2) (2005), 343-353.

[13] R. Aghatehrani and R. Kavasseri, Sensitivity-analysis-based sliding mode control for voltage regulation in microgrids, IEEE Trans on Sustainable Energy 4(1) (2013), 50-57.

[14] S.H. Li, M.M. Zhou and X.H. Yu, Design and implementation of terminal sliding mode control method for PMSM speed regulation system, IEEE Trans on Industrial Informatics 9(4) (2013), 1879-1891.

[15] Y. Shtessel, C. Edwards, L. Fridman and A. Levant, Sliding mode control and observation, Springer, New York, 2014. 